\title{
Difficult Endoscopic Retrieval of a Migrated Stent inside a Pseudocyst
}

\author{
Lien-Fu Lin Jai-Nien Tung \\ Division of Gastroenterology, Department of Internal Medicine, Tung's Taichung \\ Metroharbor Hospital, Taichung, Taiwan, ROC
}

\section{Key Words}

Pseudocyst of the pancreas - Stent - Endoscopic ultrasound guided cystogastrostomy . Forceps

\begin{abstract}
Endoscopic ultrasound (EUS) guided pseudocyst drainage can have complications such as bleeding, perforation, infection, and stent migration. We report a case of iatrogenic migration of stent inside a large dumbbell-shaped pseudocyst managed successfully with a difficult endoscopic retrieval procedure.
\end{abstract}

\section{Introduction}

Endoscopic ultrasound (EUS) guided drainage can be regarded as the treatment of choice or as an alternative to surgery in symptomatic pancreatic pseudocyst $[1,2]$. The incidence of migration of stents into the pseudocyst can reach $3 \%$ [3]. The retrieval of the migrated stent inside the pseudocyst should not be difficult as long as the cystogastrostomy opening is large enough for the endoscope to enter the pseudocyst which has no deformity. We herein report the difficult endoscopic retrieval of a migrated stent inside a large dumbbell-shaped pseudocyst.

\section{Case Report}

A 30-year-old man was admitted to our hospital in November 2007 due to a fall from a 15-foot height with bilateral radial fractures. The initial abdominal computerized tomography did not reveal any intra-abdominal injury, but he was found to have fluid collection at the tail of the pancreas 2 days later by ultrasound examination. Serum amylase level was $900 \mathrm{U} / \mathrm{l}$ (reference 36-128 U/l), and he was treated conservatively with parenteral nutrition as well as for pain. Four weeks after hospitalization, the pseudocyst increased in size, measuring $17 \mathrm{~cm}$ in it largest diameter, and the patient became symptomatic with pain and fever. ERCP revealed no pancreatic leakage. EUS guided cystogastrostomy (Olympus GF UCT 2000, EUC 2000) was done under fluoroscopy with conscious sedation (pethidine 
$50 \mathrm{mg}$ intramuscularly and midazolam $5 \mathrm{mg}$ intraveneously). The pseudocyst was punctured under EUS guidance with a 19-G needle (Wilson Cook echo-19), dilated with an 8-mm-sized balloon, and 2 double pig-tail stents were inserted (one 8.5 Fr and one $10 \mathrm{Fr}$ ). While inserting the second stent, the first stent was accidentally pushed inside the pseudocyst (fig. 1). The patient's pain and fever subsided for 1 day but recurred on the 3rd day. The migrated stent was not retrieved on the first occasion because a second 10 -Fr-sized stent was still working. It was also anticipated that the size of the large pseudocyst would get smaller, making it easier to retrieve the migrated stent at a later date. Furthermore, the patient was getting irritable with the prolonged procedure.

A second intervention was then carried out on the 3rd day. The cystogastrostomy opening (gastric wall and pseudocyst wall) was dilated with a 15-mm-sized balloon. The endoscope (Olympus GIF XQ260) was inserted into the pseudocyst cavity at the same time, suctioning the fluid and debris inside the pseudocyst. A transparent cap at the tip of the endoscope was not loaded since it would not have been of much help as the aim in this case was to retrieve the stent inside the large pseudocyst cavity. The migrated stent was located beyond the narrow neck of the dumbbell-shaped pseudocyst which was far from the cystogastrostomy opening, with the other non-migrated stent bridging the narrow neck of the pseudocyst (fig. 2e). Various methods were tried to extract the migrated stent, including forceps, basket, and balloon catheter, but all failed. The non-migrated stent bridging the narrow neck of the pseudocyst was then pulled out to provide a better view and more space. A rotatable forceps (Olympus FG-44NR-1) was used instead and the migrated stent was successfully removed under fluoroscopic and endoscopic guidance (fig. 2f, g). The retrieval procedure took about $70 \mathrm{~min}$. Reinsertion of two 10-Fr-sized double pig-tail stents was done. The pseudocyst got smaller from $17 \mathrm{~cm}$ to $2.9 \mathrm{~cm}$ in 12 days. The patient was discharged and followed up regularly.

\section{Discussion}

EUS guided transmural pseudocyst drainage is already an established effective treatment of pseudocyst of the pancreas $[1,2]$. Endoscopic clearance of the necrotic tissue in pancreatic necrosis has also been reported $[4,5]$. If the migrated stent is inside a pseudocyst without deformity, endoscopic retrieval of the stent is not a problem. In our case, the difficulties encountered were: (1) the migrated stent was beyond the narrow neck of a large dumbbell-shaped pseudocyst; (2) the visual field beyond the narrow neck was not clear; (3) the interference of a non-migrated stent bridging the narrow neck of the pseudocyst. Dilatation of the cystogastrostomy tract with a larger balloon on the first intervention would probably have prevented the occurrence of iatrogenic stent migration.

In summary, the key points to consider for successful retrieval of a migrated stent in this case include the removal of the obstacle that hindered the visual field (the non-migrated stent), combining the use of endoscopic and fluoroscopic guidance, and utilization of a rotatable forceps to facilitate retrieval. 
Fig. 1. a EUS guided needle puncture. b, c Balloon dilatation (size $8 \mathrm{~mm}$ ). d After first double pig-tail stent insertion. $\mathbf{e}$ The first stent was pushed into the pseudocyst after the 2 nd stent insertion. $\mathbf{f}$ The migrated stent inside the pseudocyst.
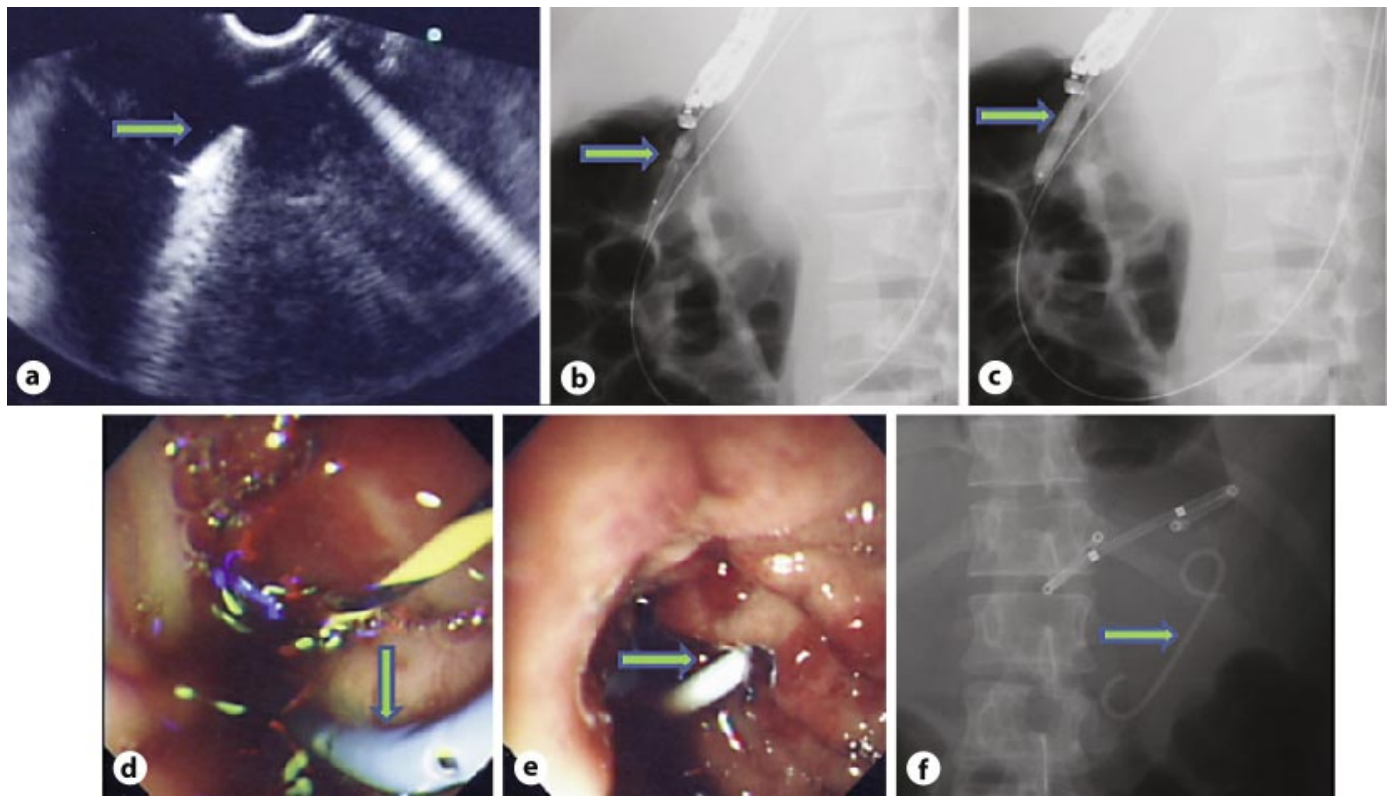

Fig. 2. a, b Second intervention (after 2 days) with 15-mm balloon dilatation of the cystogastrostomy opening (green arrows), and the inwards migrated stent (white arrow). c The endoscope entering the pseudocyst cavity. $\mathbf{d}$ The interior of the pseudocyst. $\mathbf{e}$ The migrated stent was located beyond a narrow neck (green arrow) of the pseudocyst, with the other non-migrated stent bridging the neck (white arrow). $\mathbf{f}$ A better view and space for retrieval of the migrated stent after removal of the bridging non-migrated stent. $\mathbf{g}$ Retrieval of the migrated stent under endoscopic and fluoroscopic guidance. $\mathbf{h}$ The extracted migrated stent.
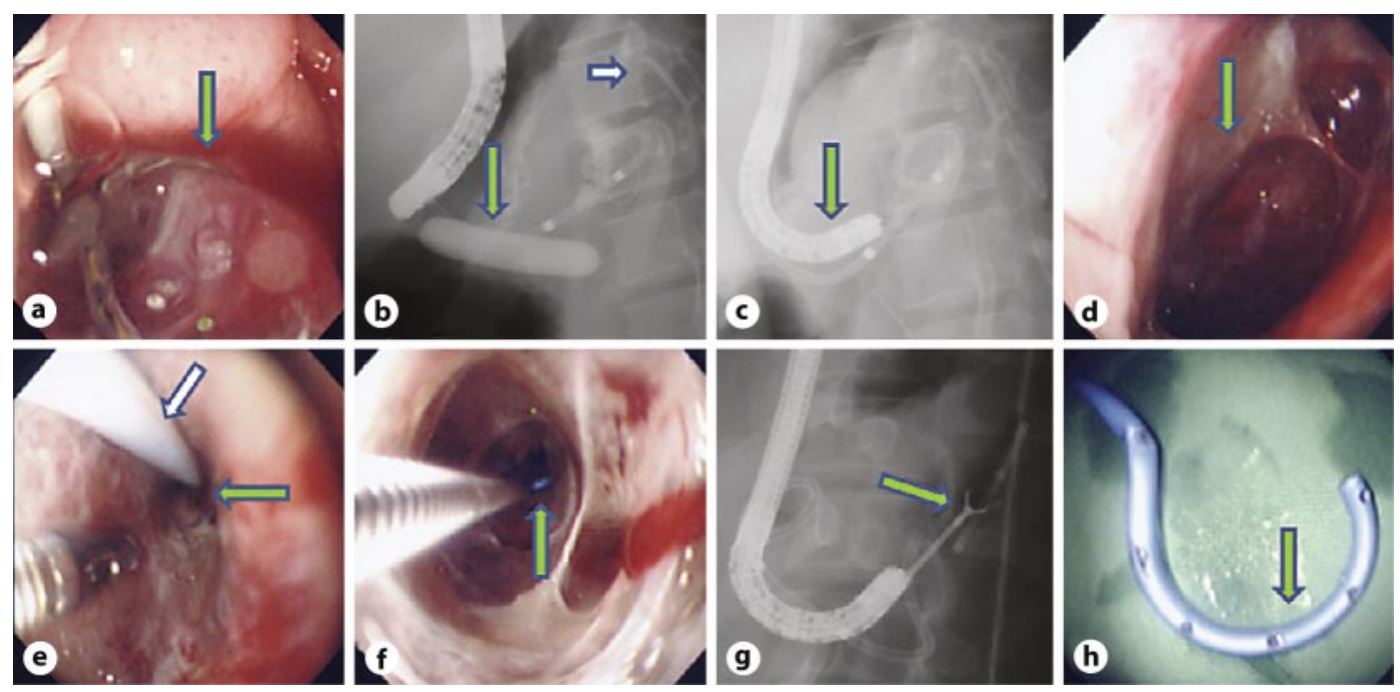


\section{References}

1 Fockens P: EUS in drainage of pancreatic pseudocysts. Gastrointest Endosc 2002;6(suppl):S93-S97.

2 Vosoghi M, Sial S, Garrett B, Feng J, Lee T, Stabile BE, Eysselein VE: EUS-guided pancreatic pseudocyst drainage: review and experience at Harbor-UCLA Medical Center. Med Gen Med 2002;4:2-6.

-3 Kahaleh M, Shami VM, Conaway MR, Tokar J, Rockoff T, De La Rue SA, de Lange E, Bassignani M, Gay S, Adams RB, Yeaton P: Endoscopic ultrasound drainage of pancreatic pseudocyst: a prospective comparison with conventional endoscopic drainage. Endoscopy 2006;38:355-359.

4 Seewald S, Groth S, Omar S, Imazu H, Seitz U, de Weerth A, Soetikno R, Zhong Y, Sriram PV, Ponnudurai R, Sikka S, Thonke F, Soehendra N: Aggressive endoscopic therapy for pancreatic necrosis and pancreatic abscess: a new safe and effective treatment algorithm. Gastrointest Endosc 2005;62:92-100.

-5 Voermans RP, Veldkamp MC, Rauws EA, Bruno MJ, Fockens P: Endoscopic transmural debridement of symptomatic organized pancreatic necrosis. Gastrointest Endosc 2007;66:909-916. 